Full length article

\title{
Human tendon-derived cell sheets created by magnetic force-based tissue engineering hold tenogenic and immunomodulatory potential
}

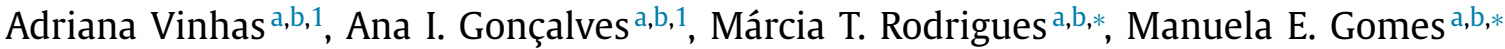 \\ a 3B's Research Group, I3Bs - Research Institute on Biomaterials, Biodegradables and Biomimetics, University of Minho, Headquarters of the European \\ Institute of Excellence on Tissue Engineering and Regenerative Medicine, Avepark - Zona Industrial da Gandra, 4805-017 Barco, Guimarães, Portugal \\ bICVS/3B's - PT Government Associate Laboratory, Braga/Guimarães, Portugal
}

\section{A R T I C L E I N F O}

\section{Article history:}

Received 19 March 2021

Revised 14 June 2021

Accepted 22 June 2021

Available online 27 June 2021

\section{Keywords:}

Tendon derived cells

Magnetic nanoparticles

Magnetic cell sheets

Inflammation

\begin{abstract}
A B S T R A C T
Cell sheet technology and magnetic based tissue engineering hold the potential to become instrumental in developing magnetically responsive living tissues analogues that can be potentially used both for modeling and therapeutical purposes. Cell sheet constructions more closely recreate physiological niches, through the preservation of contiguous cells and cell-ECM interactions, which assist the cellular guidance in regenerative processes.

We herein propose to use magnetically assisted cell sheets (magCSs) constructed with human tendonderived cells (hTDCs) and magnetic nanoparticles to study inflammation activity upon magCSs exposure to IL- $\beta$, anticipating its added value for tendon disease modeling.

Our results show that IL- $\beta$ induces an inflammatory profile in magCSs, supporting its in vitro use to enlighten inflammation mediated events in tendon cells. Moreover, the response of magCSs to IL-1 $\beta$ is modulated by pulsed electromagnetic field (PEMF) stimulation, favoring the expression of anti-inflammatory genes, which seems to be associated to MAPK(ERK1/2) pathway. The anti-inflammatory response to PEMF together with the immunomodulatory potential of magCSs opens new perspectives for their applicability on tendon regeneration that goes beyond advanced cell based modeling.
\end{abstract}

\section{Statement of significance}

The combination of cell sheets and magnetic-based technologies holds promise as instrumental bioinstructive tools both for tendon disease modelling and for the development of magnetically responsive living tendon substitutes. We have previously shown that remote actuation of a pulsed electromagnetic field (PEMF) modulated the inflammatory response of IL- $1 \beta$-treated human tendon-derived cell (hTDCs) monolayers. As magnetic cell sheets (magCSs) technologies enable improved cellular organization and matrix deposition, these constructions could better recapitulate tendon niches. In this work, we aimed to apply magCSs technologies to study hTDCs responses in inflammatory environments. Overall results show that PEMF-stimulated-magCSs hold evidence for immunomodulatory properties and to become a living tendon model envisioning tendon regenerative therapies.

(c) 2021 Acta Materialia Inc. Published by Elsevier Ltd. All rights reserved.

\section{Introduction}

Cell sheet technology introduces the possibility of providing stable cellular constructs and thus enabling the construc-

\footnotetext{
* Corresponding authors.

E-mail addresses: mrodrigues@i3bs.uminho.pt (M.T. Rodrigues), megomes@i3bs.uminho.pt (M.E. Gomes).

1 Equally contributed.
}

tion of tissue-like assemblies [1]. In fact, cell sheets (CSs) preserve structural elements as ion channels, growth factor receptors, and cell surface proteins as well as intercellular connections and matrix components, which assist cell sheet usefulness either as biomimetic models to address tissue functional aspects or as tissue substitutes. Previous studies reported cell sheet potential to stimulate musculoskeletal tissue regeneration [2,3]. Furthermore, mesenchymal stem cell sheets demonstrated a satisfactory integration into native tissues accompanied by anti-inflammatory ef- 
fects improving cartilage repair and treatment of osteoarthritis disease [4,5]. With inflammation increasingly recognized as a central component both in healing and in disease progression, a model to study inflammation in tendon tissues taking advantage of CSs technology could become an important tool to advance knowledge in this field. For instance, it has been reported that adipose tissue derived stem cells (ASC) sheets improve matrix remodeling, and modulate the inflammatory phase facilitating tendon healing [6].

Magnetic cell sheets (MagCSs) were constructed by magnetic force-based TE (Mag-TE) as firstly proposed by Ito A. et al. in 2004 [7], and described by our group [2]. Specifically, this methodology uses magnetite nanoparticles and magnetic force to construct magnetic sheets. As so, a confluent monolayer of cells is combined with magnetic nanoparticles (MNPs) and cultured under a permanent magnet. MagCSs offer the opportunity to study inflammatory cues and cell responses through contact-free stimulation of tendon resident cells in a more tissue-like environment with intercellular signaling and structural complexity [5,8]. Also, magnetic cell sheets technology has demonstrated promising results in vascular $[9,10]$, cardiac $[11]$ and musculoskeletal $[2,12,13]$ tissue engineering strategies, however, very few MagCSs examples were provided in the literature for therapies aiming at resolving inflammation. Our group has previously shown that magCSs exhibited a tendon-like extracellular matrix (ECM), good mechanoelastic properties and responsiveness, suggesting the applicability of these living patches in tendon therapies [2]. Morever, magCS could also enable to insight on pulsed electromagnetic field (PEMF) assisted immunomodulatory effects in cell-based therapies for functional tendon replacement.

A controlled pro-inflammatory milieu can be provided to magnetic tendon cell sheets by the exogenous supplementation of interleukin- $\beta$ (IL- $1 \beta$ ) to the culture medium. IL- $1 \beta$ is a proinflammatory cytokine released in inflammatory environments [14] and a well described marker of chronic inflammation [15,16]. From previous reports by our group $[17,18]$ and others [19-21] exposure to low frequency ( 2 to $75 \mathrm{~Hz}$ ) and low strength (1.5 to $82 \mathrm{mT}$ ) magnetic fields, was shown to modulate tendon cell response to inflammation stresses influencing the expression and release of cytokines $[17,18]$. Other works also showed the effectiveness of the combination of MNPs with magnetic field for pain control and enhanced functional recovery in the knee diseases and osteoarthritic lesions [22]. Moreover, it has been reported by Rahmi et al. [23] that bone marrow-derived mesenchymal stem cell sheets labelled with MNPs upregulated anti-inflammatory factors, TGF- $\beta 2$ and IL-10, which increases the potential of magnetic cell sheets for therapeutic strategies.

In this work, we aimed at using magnetic cell sheet technologies to study tendon cell responses in inflammatory environments, induced by exposure of the cell sheets to IL- $1 \beta$. We propose magCSs as a pathophysiological model of inflammation to more closely study the onset of tendon lesions and the inflammatory molecules affecting injured tendons, taking advantage of magCSs properties over cell monolayers. Furthermore, as PEMF favors anti-inflammatory cell responses, we also studied the role of magCSs assisted by a remote PEMF in IL- $1 \beta$ induced tendon cells responses.

\section{Materials and methods}

\subsection{Isolation and culture of human tendon derived cells (hTDCs)}

hTDCs were isolated from surplus healthy tissue samples of patellar tendons, collected from adult patients undergoing orthopedic reconstructive surgeries under protocols previously established with Hospital da Prelada (Porto, Portugal) and with informed consent of the patients. The content of the written informed consent and related procedures were reviewed and approved by the Hospital Ethics Committee (P.I. N. $\left.{ }^{\circ} 005 / 2019\right)$. The tendon tissue identification and quality (healthy/diseased) was assessed by the medical team during surgical intervention.

Following a previously established protocol [24-26], tendon samples were minced using a sterile scalpel. Phosphate buffer saline (PBS) drops were added in a continuous basis to keep a moist environment and reduce cell damage by mechanical forces. The excess of PBS was removed using a filtration system for $50 \mathrm{ml}$ tubes (Falcon). Minced samples were collected into a $50 \mathrm{~mL}$ tube, already containing an enzymatic solution of collagenase $(0.1 \%$, Sigma-Aldrich, C6885, USA) with $2 \mathrm{M} \mathrm{CaCl2} \mathrm{(1:1000,} \mathrm{VWR,} \mathrm{Ger-}$ many) and $1 \%$ bovine serum albumin (BSA) (Sigma-Aldrich, USA) for $1 \mathrm{~h}$ at $37^{\circ} \mathrm{C}$ under constant agitation. A ratio of $1: 1$ of minced tissue to the enzymatic solution was considered. After incubation, digested samples were filtered and centrifuged three times at $290 \mathrm{~g}$ for $5 \mathrm{~min}$, and the supernatant discarded. Isolated hTDCs were expanded in $\alpha$-MEM medium (A-MEM, Invitrogen, Life Technologies Limited, Paisley, UK) composed of $\alpha$-MEM supplemented with $10 \%$ fetal bovine serum (FBS) (Alfagene, Life Technologies Limited, UK) and $1 \%$ antibiotic/antimicotic solution (A/A) (Alfagene, Life Technologies Limited, UK) in humidified $5 \% \mathrm{CO}_{2}$ atmosphere. hTDCs from passage 1 to 3 were used to perform all the experiments.

\subsection{Magnetic cell sheets construction, culture, and stimulation}

Magnetic cell sheets (magCSs) were constructed as previously described by our group [2]. Briefly, hTDCs were seeded onto 24well tissue culture plates (BD Biosciences, San Jose, UK) at a density of 500,000 cells per well. Two hours after seeding, the chitosan coated iron oxide MNPs (nanomag-C, 04-00-152, Micromod, Germany) were added to adherent hTDCs at $200 \mathrm{pg} / \mathrm{cell}$. MNPs were overnight incubated with the cells in $\alpha$-MEM medium (AMEM, Invitrogen, Life Technologies Limited, Paisley, UK) on a 24well permanent magnet well-array plate (magnetic induction of $350 \mathrm{mT}$ per magnet per well) in humidified $5 \% \mathrm{CO}_{2}$ atmosphere. Then, $16 \mathrm{~h}$ after MNPs addition, magCSs were washed with D-PBS (Sigma-Aldrich, Saint Louis, USA) and the culture medium replaced by either i) $\alpha$-MEM medium (magCSs Control group) or ii) $\alpha$-MEM medium supplemented with IL- $1 \beta(1 \mathrm{ng} / \mathrm{mL}$, Alfagene, Life Technologies Limited, UK) for $24 \mathrm{~h}$ (magCSs IL- $1 \beta$ group) to induce inflammatory cues in hTDCs, as previously established [17]. To assess the immunomodulatory effect of PEMF, $24 \mathrm{~h}$ after supplementation with IL- $1 \beta$ magCSs were stimulated with PEMF (magCSs IL-1 $\beta$ PEMF group) using a magnetotherapy device (Magnum XL Pro; Globus Corporation, Italy) for $1 \mathrm{~h}$ in humidified $5 \% \mathrm{CO}_{2}$ atmosphere. The magCSs were placed between two coils (solenoids) and the magnetic field was generated by the electric current that goes through a coil.

The stimulus provided was selected from a set of therapeutic programs used in health-rehab medical treatments. These treatments typically range between $10 \mathrm{~min}$ and $12 \mathrm{~h}$ and the parameters of magnetic fields range between 5 to $200 \mathrm{~Hz}$, and strength 0.2 to $10 \mathrm{mT}$. These parameters are known to be safe and with therapeutic value in humans. Having this in mind, together with the outcomes from previous studies of our group with tendon cell cultures [17], the PEMF parameters of $5 \mathrm{~Hz}$ of frequency, $4 \mathrm{mT}$ of intensity and $50 \%$ duty cycle were established as optimal parameters for modulating inflammatory cues. In these studies we also determined the $1 \mathrm{~h}$ exposure period as suffice to elicit a desirable response in IL- $1 \beta$-primed tendon cells either cultured in monolayer [17] or in magnetic-responsive systems [27].

In sum, the following conditions were investigated: i) magCSs in $\alpha$-MEM medium (Control), ii) IL- $1 \beta$-treated magCSs (IL- $1 \beta$ ), iii) IL- $1 \beta$-treated magCSs exposed to PEMF (IL- $1 \beta$ PEMF). Magnetic 
cell sheets were characterized at day 3 (counting from tendon cell seeding on the plates) for cell viability, proliferation and morphological analysis. Matrix deposition, collagen production, and gene expression of tendon and inflammatory markers were also investigated. Additionally, the culture media of magCSs was collected and the presence of inflammatory mediators quantified using ELISA assays.

\subsection{Cell distribution and matrix deposition - tendon cell monolayers vs magCSs constructions}

A Hematoxylin-Eosin (HE) stain was performed for comparative analysis of the spatial distribution of cells in tendon cell monolayers and magCSs constructions. Briefly, tendon cell monolayers and magCSs (control condition), both seeded at 500,000 cells/well and cultured for 3 days were stained using Hematoxylin (7211, Thermo Scientific, USA) and Eosin-Y Alcoholic solution (71204, Thermo Scientific, USA).

To confirm the stability and matrix deposition in magCSs, a Sirius Red/Fast Green Collagen Staining Kit (9046, Chondrex, Woodinville, USA) was performed, and results compared to tendon cell monolayers. magCSs and tendon cell monolayers were fixed with a $10 \%(\mathrm{v} / \mathrm{v})$ neutral buffered formalin solution (Bio Optica, Milano, Italy). Then the dye solution from the kit was added to the magCSs and to the cells in monolayer followed by $40 \mathrm{~min}$ incubation.

HE and Sirius Red/Fast Green stained samples were visualized and images obtained using a fluorescence inverted microscope (Axio Observer, Zeiss, Göttingen, Germany).

Immunostaining for collagen I was also performed as detailed described in Section 2.9.

\subsection{Assessment of cellular viability and cell proliferation in magCSs constructions}

Live/dead assay was performed to assess cellular viability. Briefly, the magCSs were incubated for $20 \mathrm{~min}$ with calcein-AM $(2 \mu \mathrm{g} / \mathrm{ml})$ and propidium iodide $(3 \mu \mathrm{g} / \mathrm{ml})$, both from Life Technologies Limited, UK. Afterwards, cells were washed with PBS and visualized under a fluorescence inverted microscope (Axio Observer, ZEISS, Germany).

The cell viability of magCSs was evaluated by MTS assay (Cell Titer 96 Aqueous Solution Cell Proliferation Assay, Promega). MagCSs were washed in PBS before a $3 \mathrm{~h}$ incubation in a mixture of phenol red-free medium supplemented with FBS and MTS solution (5:1 ratio) at $37{ }^{\circ} \mathrm{C}$ and $5 \% \mathrm{CO}_{2}$ atmosphere, protected from light. Then, the supernatant was transferred to a new 96-well plate and the absorbance read at $490 \mathrm{~nm}$ (Synergy ${ }^{\mathrm{TM}} \mathrm{HT}$, BIO-TEK Instruments, Winooski, USA). Triplicates were made of each sample and a blank sample (no cells) reading was performed.

The cell proliferation of magCSs constructions were evaluated by Quant-It PicoGreen dsDNA assay Kit (Thermo Fisher Scientific, USA). magCSs from all conditions were washed with PBS, transferred to a microtube with $1 \mathrm{ml}$ of sterile ultrapure water and kept at $-80{ }^{\circ} \mathrm{C}$ until analysis. Samples were thawed, sonicated and processed according to the manufacturer's instructions, at an excitation/emission wavelength of 485/528 nm (Synergy $^{\mathrm{TM}}$ HT, BIO-TEK Instruments, Winooski, USA). Samples and standards were made in triplicate.

\subsection{Morphological characterization}

The morphological characterization of the magCSs was analyzed by scanning electron microscopy (Auriga Compact, ZEISS, Germany). The elementary composition of magCSs was also investigated by Energy dispersive x-ray spectroscopy (EDS) (JSM-6010LV, JEOL, Japan) to confirm the presence of iron (Fe) element within
magCSs constructions. For this purpose, the magCSs were fixed in $10 \%(\mathrm{v} / \mathrm{v})$ neutral buffered formalin for $1 \mathrm{~h}$ at room temperature and dehydrated in a series of increasing ethanol solutions (from $30 \%$ to $100 \%(\mathrm{v} / \mathrm{v})$ ), followed by a $5 \mathrm{~min}$ immersion in hexamethyldisiloxane (HMSO, Sigma-Aldrich, Saint Louis, USA). Subsequently, the samples were air-dried overnight and sputter coated with gold (30 s at $20 \mathrm{~mA}$, Cressington, C5219, Model 108A, USA).

\subsection{Gene expression evaluation by RT-PCR}

The expression of tenogenic markers, namely Mohawk $(M K X)$, Scleraxis $(S C X)$, Tenomodulin (TNMD), Decorin (DCN), Metalloproteinase-1 (MMP-1), Metalloproteinase-2 (MMP-2), Metalloproteinase-3 (MMP-3), Inhibitor of Metalloproteinase-1 (TIMP-1), Collagen type I (COL1A1), Collagen type III (COL3A1), and inflammation markers, namely Interleukin-8 (IL-8), Interleukin$1 \beta$ (IL-1 $\beta$ ), Tumor necrosis factor (TNF $\alpha$ ), Interleukin-6 (IL-6), Interleukin-10 (IL-10), Interleukin-4 (IL-4), were assessed by real time RT-PCR.

Total RNA was extracted from all samples under study, using TRI reagent (T9424, Sigma-Aldrich, Saint Louis, USA) according to the manufacturer's instructions. Briefly, TRI reagent was added to each sample and stored at $-80^{\circ} \mathrm{C}$. After defrosting, samples were incubated with chloroform (Sigma-Aldrich, Saint Louis, USA) for $15 \mathrm{~min}$ and centrifuged at $12,000 \mathrm{~g}$ for $15 \mathrm{~min}$ at $4{ }^{\circ} \mathrm{C}$. The aqueous fraction was collected and isopropanol (Sigma-Aldrich, Saint Louis, USA) was added. After $10 \mathrm{~min}$, samples were centrifuged at $12,000 \mathrm{~g}$ for $10 \mathrm{~min}$ at $4{ }^{\circ} \mathrm{C}$. RNA pellet was washed with ethanol $(70 \% \mathrm{v} / \mathrm{v})$ and subsequently centrifuged at $7500 \mathrm{~g}$ for $5 \mathrm{~min}$ at $4{ }^{\circ} \mathrm{C}$. RNA quantity and purity were determined with a NanoDrop ND-1000 spectrophotometer (Wilmington, USA). The cDNA synthesis was performed using the qScript cDNA Synthesis kit (qScript ${ }^{\mathrm{TM}}$ cDNA Synthesis Kit, Quanta Biosciences, Gaithersburg, USA) in a Mastercycler Realplex (Eppendorf, Hamburg, Germany). An initial amount of total RNA of $1 \mu \mathrm{g}$ in a total volume of $20 \mu \mathrm{L}$ was used per each and every sample. The quantification of the transcripts was carried out by quantitative polymerase chain reaction (qPCR) using the PerfeCTA SYBR Green FastMix kit (Quanta Biosciences, Gaithersburg, USA) following the manufacturer's protocol, in a Real-Time Mastercycler Realplex thermocycler (Eppendorf, Hamburg, Germany).

The primers were pre-designed with PerlPrimer v1.1.21 software (Supplementary Table S1) and synthesized by MWG Biotech. $G A P D H$ was used as the housekeeping gene. The $2^{-\Delta \Delta \mathrm{Ct}}$ method was selected to evaluate the relative expression level for each target gene. All values were firstly normalized against GAPDH expression values, and then to magCSs cultured in $\alpha$-MEM medium at day 3.

\subsection{Quantification of secreted forms of inflammatory mediators}

The release of inflammatory mediators was analysed from cell supernatants of all magCSs conditions. The supernatants were tested using commercially available enzyme immunoassay kits for TNF $\alpha$ (Human TNF $\alpha$ ELISA Kit, EK0525, Tebu-Bio, Fremont, USA), COX-2 (Human COX-2 ELISA Kit, KA0323, Abnova, Taiwan), $\mathrm{PGE}_{2}$ (Human Prostaglandin E2 ELISA Kit, KHL1701, Thermo Fisher Scientific, Molecular Probes, Eugene, USA), IL-6 (Human IL-6 Standard ABTS ELISA Development Kit, 900-K16, Peprotech, Rocky Hill, USA), and IL-10 (Human IL-10 Standard ABTS ELISA Development Kit, 900-K21; Peprotech, Rocky Hill, USA). MAPK protein phosphorylation was also determined in cytoplasmic protein extracts using the PhosphoTracer ERK1/2 (pT202/Y204)+p38 MAPK (pT180/Y182)+JNK1/2/3 (pT183/Y185) Elisa Kit (Abcam, ab119674, Cambridge, UK). Each and every kit were performed following the manufacturers' instructions. 


\subsection{Quantification of extracellular matrix proteins}

The amount of collagen and non-collagenous proteins was determined using a semiquantitative assay, namely Sirius Red/Fast Green Collagen Staining Kit (9046, Chondrex, Woodinville, USA). For this purpose, samples of all magCSs conditions were fixed with a neutral buffered formalin solution and stored at $4{ }^{\circ} \mathrm{C}$ until analysis. Then the dye solution from the kit was added to the magCSs followed by 40 min incubation. A dye extraction buffer was then mixed and the OD values read in a spectrophotometer (Synergy HT, Biotek Instruments) at $540 \mathrm{~nm}$ and $605 \mathrm{~nm}$. The results of collagen and non-collagenous proteins were normalized to dsDNA quantified in Section 2.4.

\subsection{Evaluation of tenogenic markers in magCSs constructions}

Protein expression of Mohawk, Tenomodulin and Collagen type I was assessed by immunocytochemistry for all magCSs conditions in study. magCSs were washed three times with PBS, before and after fixation with $10 \%(\mathrm{v} / \mathrm{v})$ neutral buffered formalin overnight and kept in PBS at $4{ }^{\circ} \mathrm{C}$ until usage. Subsequently, the cells were incubated with $0.025 \%$ Triton-X100 in PBS solution (Sigma-Aldrich, Saint Louis, USA) and the blocking step was performed using Normal Horse Serum (Ready To Use Vectastain Kit, PK-7200; Vector, California). The magCSs were incubated overnight with anti-MKX (Rabbit anti-human, A83377, 1:100, Sigma-Aldrich, Saint Louis, USA), anti-Tenomodulin (Rabbit anti-human, ab81328, 1:100, Abcam, Cambridge, UK), anti-Collagen type I (Rabbit antihuman, ab34710, 1:100, Abcam, Cambridge, UK) diluted in antibody diluent with background reducing components (Dako, Santa Clara, USA) at $4{ }^{\circ} \mathrm{C}$, followed by $1 \mathrm{~h}$ incubation at room temperature with the secondary antibody (donkey anti-rabbit Alexa Fluor 488, 1:200; Alfagene, Life Technologies Limited, UK). The samples were washed three times with PBS and stained with $4^{\prime}, 6$ diamidino-2-phenyindole, dilactate (DAPI, $5 \mu \mathrm{g} / \mu \mathrm{l}$, D9564; SigmaAldrich, Saint Louis, USA) for $10 \mathrm{~min}$.

The immunostained samples were then analyzed by confocal laser scanning microscopy (CLSM, TCS SP8, Leica, Wetzlar, Germany). Images were bidirectionally scanned at $400 \mathrm{~Hz}$ with Argon (488) and UV (405) lasers and acquired using a 63x magnification objective. Three independent experiments were investigated for protein detection by immunofluorescence.

\subsection{Assessment of nuclei elongation ratio}

Nuclei aspect ratio was determined measuring a minimum of 30 DAPI-stained nuclei from confocal microscopy images obtained in Section 2.9. Nuclei were imaged in different regions of magCSs samples. All three magCSs conditions were analysed using ImageJ software (version $1.52 \mathrm{~d}$ ). The elongation ratio was defined by nuclei length divided by nuclei width.

\subsection{Western blotting analysis}

The MAPK protein phosphorylation was also investigated by western blotting assay. magCSs were collected in PBS, resuspended, and the supernatant collected to a new microtube upon magnetic separation (MPC®-S, Dynal Biotech).

The total cell lysates were prepared using RIPA buffer (SigmaAldrich, Saint Louis, USA) with protease inhibitor cocktail (SigmaAldrich, Saint Louis, USA) and phosSTOP (ROCHE, Switzerland). The samples were centrifuged for $15 \mathrm{~min}$ at $21,952 \mathrm{~g}$ at $4{ }^{\circ} \mathrm{C}$ and the supernatants collected. Afterwards, the BCA protein assay kit (Alfagene, Life Technologies Limited, UK) was used to assess the protein content of the samples according to the manufacturer's instructions. The protein extracts were resolved in $10 \%$ sodium do- decyl sulfate-polyacrylamide gels, followed by semi-dry transfer to Amersham ${ }^{\mathrm{TM}}$ Protran ${ }^{\circledR}$ Western blotting membranes (nitrocellulose; Sigma-Aldrich, Saint Louis, USA). The transfer of the proteins to the membrane was performed in a Pierce Power Station (Thermo Fisher Scientific, Molecular Probes, Eugene, OR). The membranes were blocked with 5\% BSA in Tris-buffered saline with Tween 20 (Sigma-Aldrich, Saint Louis, USA) (TBS-T) and incubated with rabbit anti-phospho-p44/42 MAPK (Erk1/2) (1:1000) (9102; Cell signaling Technology, Danvers, MA), anti-collagen III (1:1000) (ab184993; Abcam, UK), anti-Tenomodulin (1:1000) (ab184993; Abcam, UK), anti-MKX (1:1000) (HPA006927; Sigma-Aldrich, Saint Louis, USA) and anti- $\alpha$ smooth muscle actin (1:2000) (ab32575; Abcam, UK) antibodies for $1 \mathrm{~h}$ at room temperature under mild agitation. The membranes were washed three times with TBS-T and then incubated with a secondary antibody (1:2000) (AntiRabbit IgG Alkaline Phosphatase antibody, A9919, Sigma-Aldrich, Saint Louis, USA) for $1 \mathrm{~h}$ at RT. A colorimetric AP substrate reagent kit (1,706,432; BioRad, Hercules, USA) was used for color development. Semi-quantitative analysis was performed for pMAPK bands using Image software (version $1.52 \mathrm{~d}$ ). $\alpha$-actin was used as an internal control and for the normalization of band measurements.

\subsection{Statistical analysis}

Results are expressed as mean \pm standard error of the mean (SEM). The statistical analysis was performed using GraphPad Prism6 software. Data was obtained from 3-independent experiments $(n=3)$ analyzed in triplicate, and evaluated by Two-way ANOVA followed by Bonferroni post-hoc test for multiple comparison tests.

Symbols denote a different degree of confidence, ${ }^{*} p<0.05$, ** $p<0.01,{ }^{* * *} p<0.001$ and ${ }^{* * * *} p<0.0001$.

\section{Results}

\subsection{Improved tendon cell organization and matrix production in} magCSs constructions

HE stain was performed to infer on the cellular distribution of magCSs in comparison to a simple tendon cell monolayer. A hyperdense culture was observed in both cultures (Fig. 1A, 1B) however cells in tendon cell monolayer are more randomly distributed. Also, magCSs evidence a higher level of spatial organization.

Sirius red/Fast green staining together with collagen I confirmed matrix deposition by tendon cells, which is more abundant and richer in collagen I in magCSs constructions (Fig. 1Aii, Bii).

\subsection{Assessment of viability, cell content and micro-morphology in magCSs constructs}

The MTS and Live/Dead assays were performed to confirm the non-cytotoxic effect of MNPs on tendon cells. High cell viability outcomes were verified in all conditions studied demonstrating the suitability of the MNPs concentration applied and of magCSs constructions for in vitro cell-based approaches (Fig. 2Ai, 2Aii).

In terms of dsDNA content (Fig. 2Aiii), IL-1 $\beta$-magCSs under PEMF showed the higher dsDNA concentration among all conditions studied $(p<0.05)$.

In tendon tissues cell nuclei and overall cell morphology follows an elongated shape. Elongated nuclei display ratio superior to 1 , and, the average aspect nuclei ratio in IL- $1 \beta$-magCSs under PEMF showed the highest values $(>1)$ among the conditions studied (Fig. 2Aiv) $(p<0.0001$ in comparison to control group and $p<0.0001$ to IL- $1 \beta$-magCSs). Moreover, IL- $1 \beta$-magCSs showed the lowest nuclei aspect ratio $(p<0.05$ to control group and $p<0.0001$ 


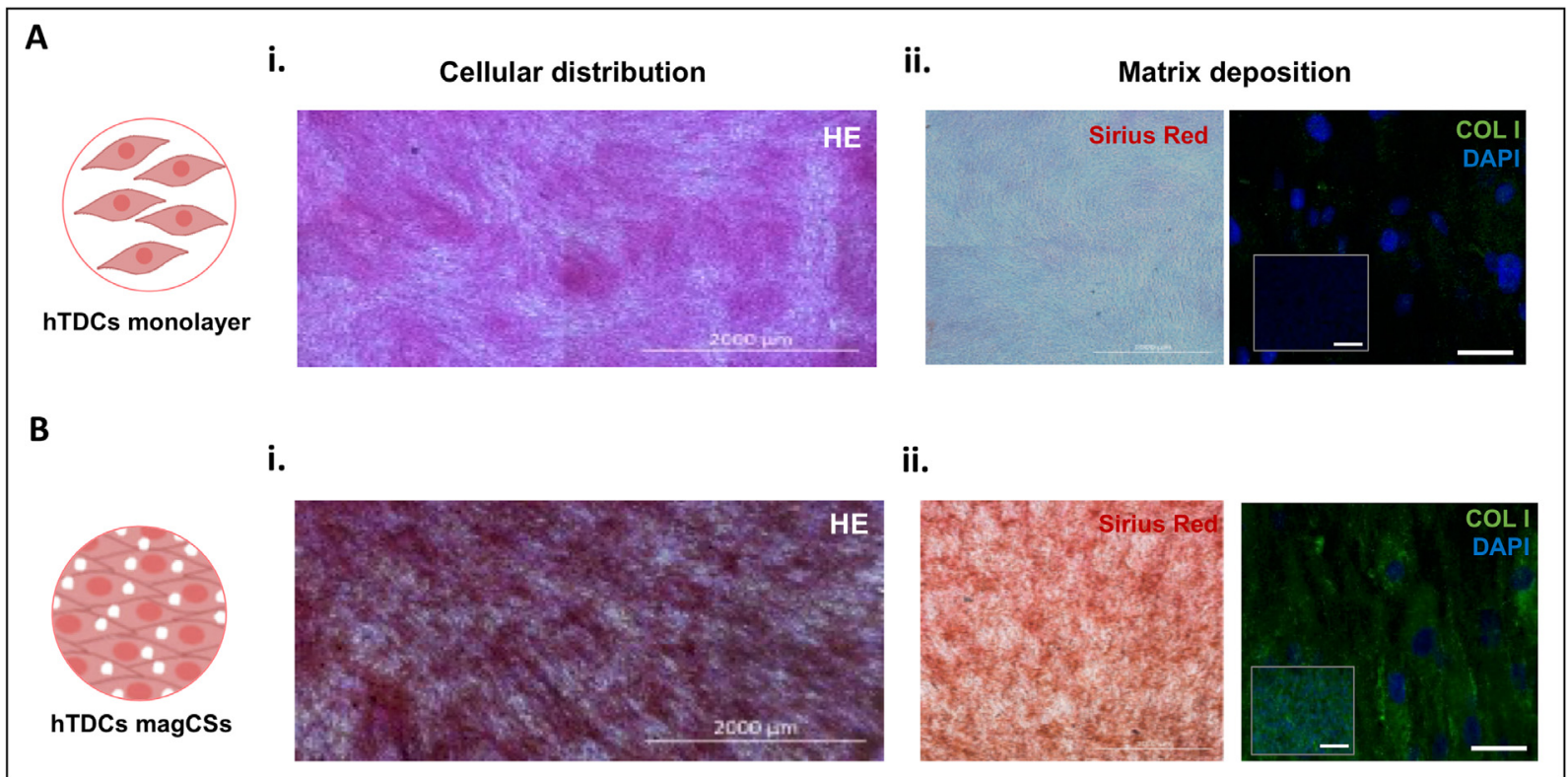

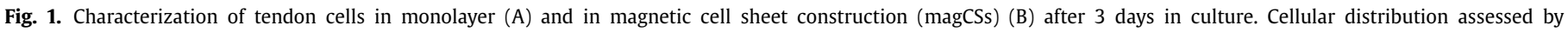

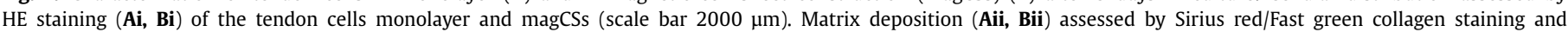

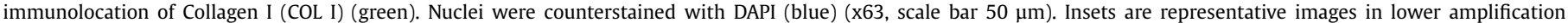
(x20, scale bar $100 \mu \mathrm{m})$.

to IL- $\beta$-magCSs under PEMF). This result suggests that PEMF favors an elongated shape nucleus, and that IL- $1 \beta$ rich environments influence a more rounded shaped nucleus.

SEM analysis (Fig. 2B) also supports a tendency for the alignment distribution of magCSs within different layers of cells suggesting the establishment of intercellular connections among cells.

\subsection{Tenogenic phenotype in magCSs under an IL-1 $\beta$ rich environment}

The impact of IL- $1 \beta$ supplementation and PEMF exposure was evaluated on the tendon markers expressed by magCSs to assess their influence on the maintenance of tenogenic profile. With an exception for $S C X$, the relative expression of $M K X, T N M D$, and $D C N$ were significantly affected by the IL- $\beta$ treatment $[19,27,28]$ (Fig. 3).

MKX and DCN show a significant decrease in the expression values in comparison to Control and IL- $1 \beta$ PEMF groups $(p<0.05)$ (Fig. 3A), while TNMD levels tend to decrease with both PEMF stimulation ( $p<0.01$ to Control) and IL- $\beta$ treatment $(p<0.0001$ to Control group) (Fig. 3A). SCX expression seems not to be affected by IL- $1 \beta$ treatment or PEMF, showing similar values for all conditions studied (Fig. 3A). The maintenance of SCX expression levels has been also observed in our previous works [27]. Overall, IL-1 $\beta$ treatment combined with PEMF stimulation leads to an increment in tendon gene expression up to the levels of non-IL- $1 \beta$ magCSs (Control group).

The presence of the tenogenic proteins MKX and TNMD was also investigated. MKX and TNMD were observed in all conditions (Fig. 3B), however the signal intensity of MKX was lower in magCSs treated with IL- $1 \beta$. Additionally, the presence of these proteins together with collagen III was confirmed by western blot analysis (Fig. 4Bii).

\subsection{Tendon-like ECM matrix in magCSs}

The normal function of tissues depends on the maintenance of the unique ECM composition and architectural arrangements.
In the particular case of tendon tissues, the matrix organization is critical to provide structure and biomechanical properties. Since we supplemented magCSs with a powerful inflammatory mediator that could contribute for architectural changes in ECM, we assessed the expression of enzymes associated to collagen degradation and to ECM remodeling phenomena.

As expected, the expression of MMP-1, MMP-2 and MMP-3 increased with IL-1 $\beta$ treatment in comparison to control group $(M M P-1, p<0.01 ; M M P-2, M M P-3 p<0.0001)$ and to magCSs exposed to PEMF (MMP-1, $\quad p<0.05 ; \quad M M P-2, \quad M M P-3, \quad p<0.0001)$ (Fig. 4Ai) [17,27]. Interestingly MMP-1 and MMP-3 levels in IL-1 $\beta$ magCSs stimulated with PEMF are higher than in control group (MMP-1, $p<0.01 ; M M P-3, P<0.0001)$. Also, the expression of TIMP-1 is upregulated in magCSs stimulated with PEMF $(p<0.0001)$, while in IL- $\beta$-magCSs was downregulated ( $p<0.0001$, in comparison to non-PEMF IL- $1 \beta$-magCSs) (Fig. 4Aii). It has been reported that increased levels of MMPs and low levels of TIMP-1 are associated to the degenerative changes in chronic tendinopathy [29]. In inflammatory conditions, elevated MMPs and decreased expression of inhibitors result in the degradation of collagens, proteoglycans and elastin fibers in the ECM [30].

In sum, the IL- $1 \beta$-magCSs respond to IL- $1 \beta$ supplementation at the gene expression level. PEMF seems to stimulate positively the expression of MKX, TNMD, DCN and TIMP-1, and inhibit MMP-1, $M M P-2$ and $M M P-3$ in IL- $\beta$-magCSs.

Collagen type $I$ is the major protein in connective tissues and an increase in collagen type III has been related both to remodeling phase of healing and to fibrotic tissues [31]. We further investigated how IL- $1 \beta$ treatment could directly affect the degradation of collagen and other proteins in ECM produced by magCSs. The expression of collagen type I (COL1A1) was decreased in IL$1 \beta$-magCSs $(p<0.0001$ in comparison to control group) but collagen type III (COL3A1) remained unchanged $(p>0.05)$ (Fig. 4Aiii).

However, in terms of collagen production, no significant differences were found between control and IL- $1 \beta$-magCSs groups $(p>0.05)$. Nevertheless, the production of non-collagenous proteins was deeply reduced in IL- $1 \beta$-magCSs $(p<0.0001$ in comparison to Control group and to IL- $1 \beta$-magCSs stimulated with PEMF group) (Fig. 4Bi). 

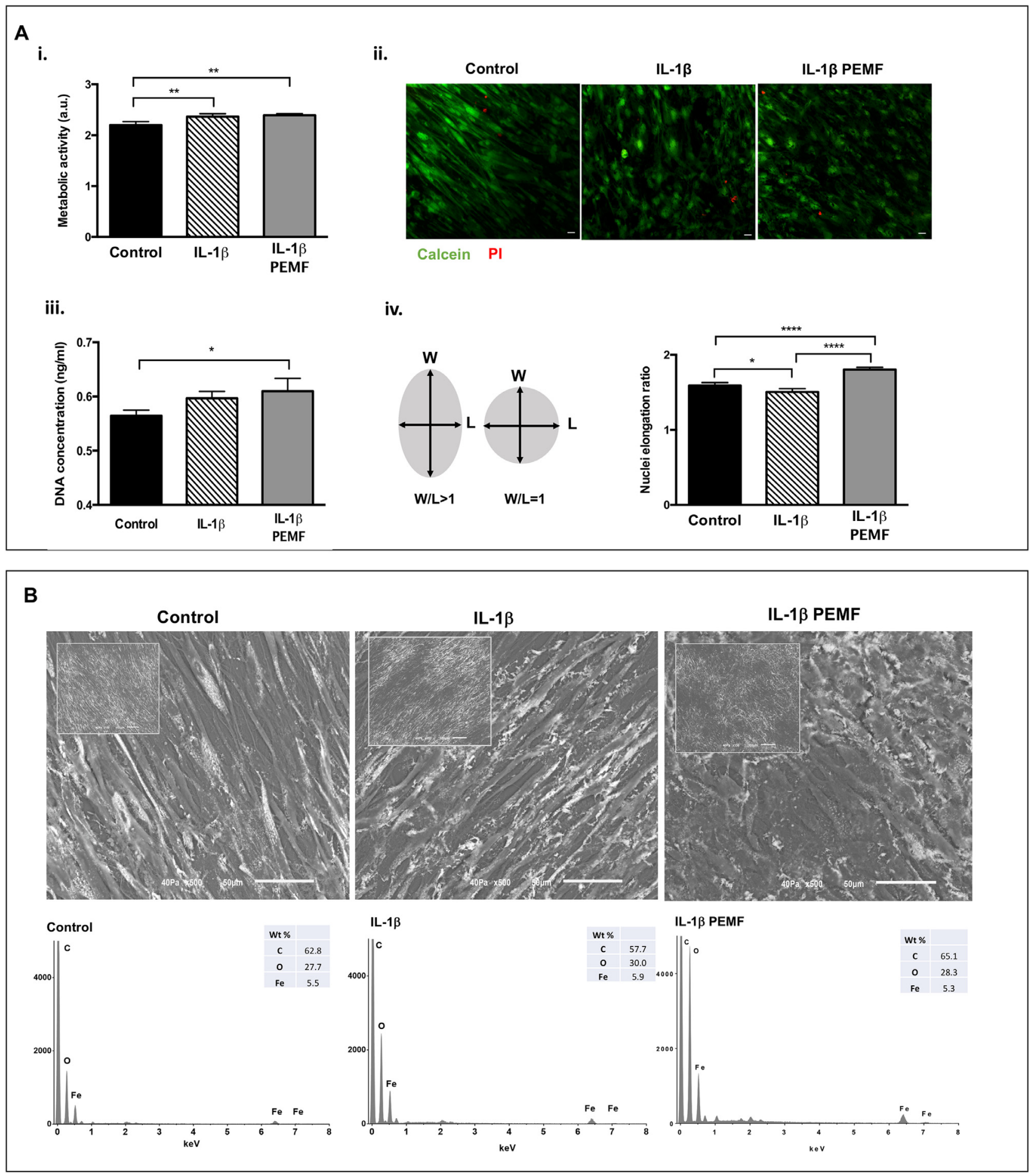

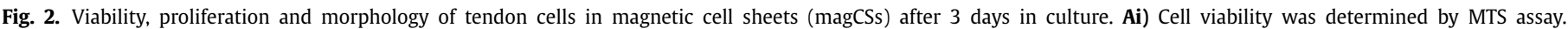

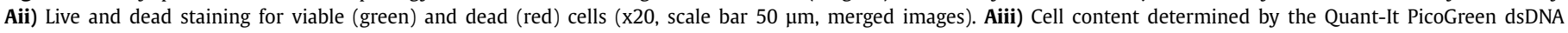

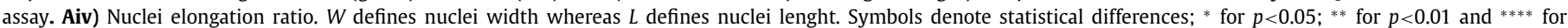

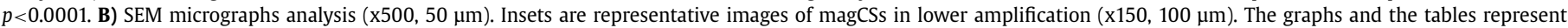

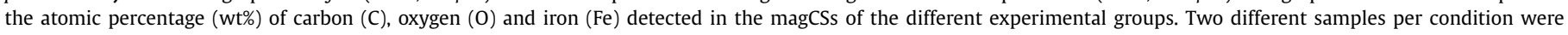

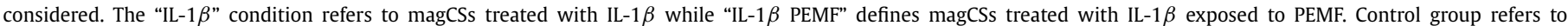
magCSs in the absence of IL- $1 \beta$ and PEMF.

When PEMF is applied on IL- $1 \beta$-magCSs, there is an increment in the COL3A1 expression $(p<0.001)$ and in the amount of both collagenous $(p<0.0001)$ and non-collagenous $(p<0.0001)$ proteins. The increment is also significant in comparison to magCSs Control group $(p<0.0001)$ (Fig. 4Bi), suggesting an anabolic role of PEMF in magCSs treated with IL- $1 \beta$ on the quality and quantity of the ECM.

Thus, PEMF seems to significantly influence the production of collagen $(p<0.05)$ in IL- $1 \beta$-magCSs. The fact that collagen synthesis is also increased in comparison to control $(p<0.05)$ group suggests 


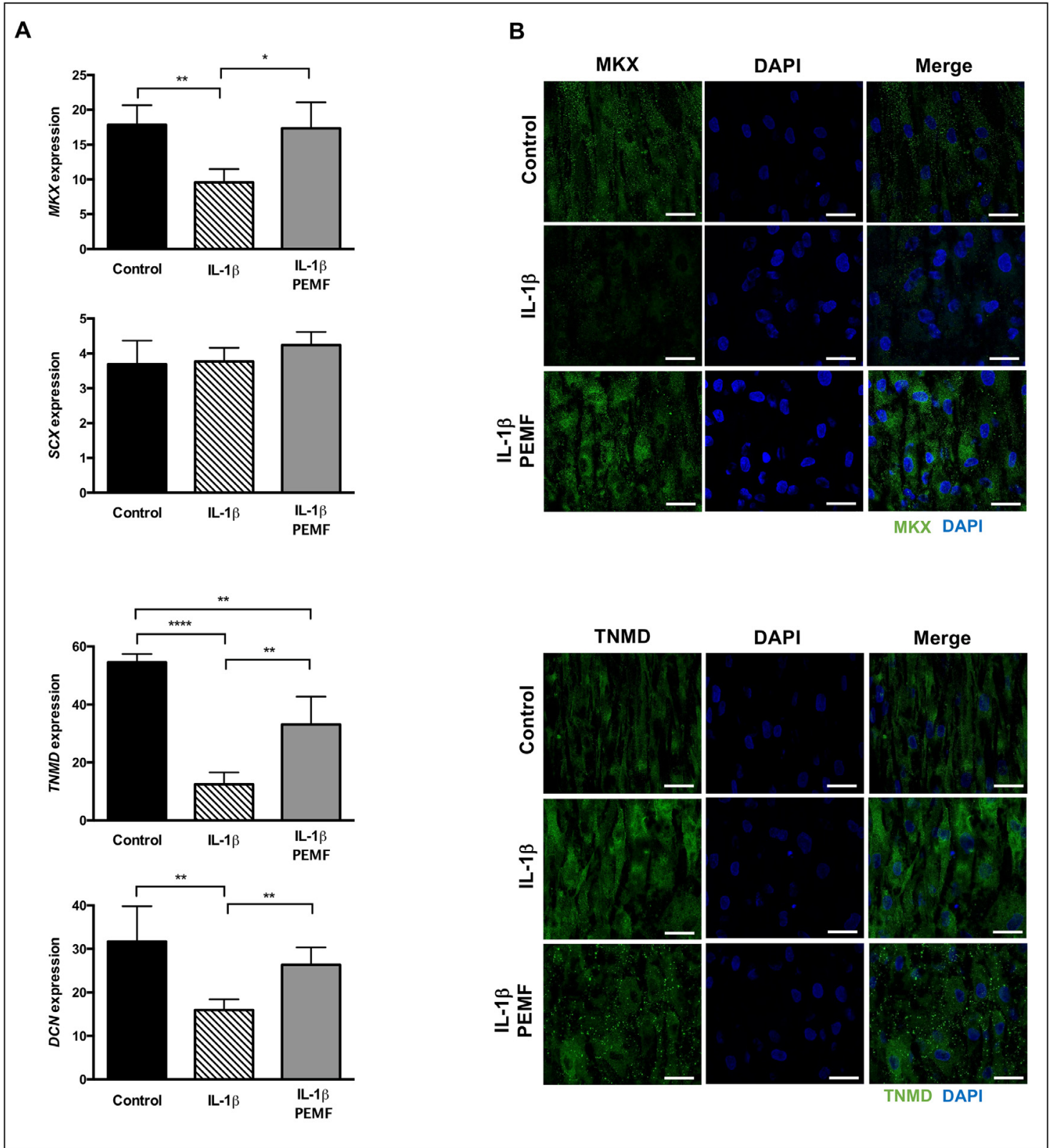

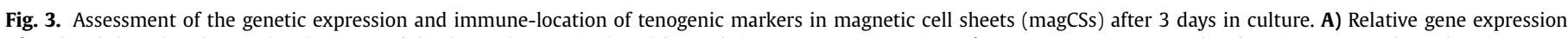

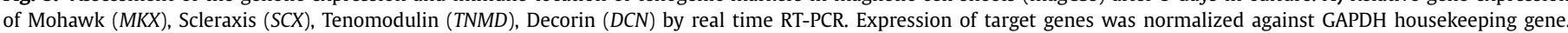

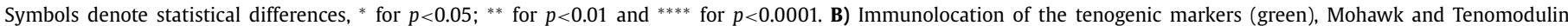

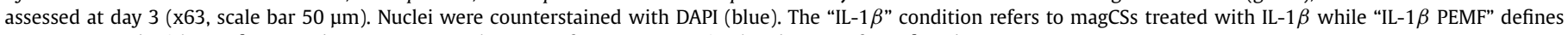
magCSs treated with IL- $1 \beta$ exposed to PEMF. Control group refers to magCSs in the absence of IL- $1 \beta$ and PEMF.

that PEMF promotes the deposition of a collagen rich-ECM matrix. Furthermore, the increased levels of COL3A1 also supports that the synthesis and deposition of collagen type I and collagen type III, necessary for the remodeling process [32].

\subsection{Investigation of cytokine profile in magCSs}

In an inflammatory milieu, cells communicate through networks of inflammatory mediators to inform about extracellular conditions and adjust the response accordingly. The treatment of magCSs with IL- $1 \beta$ was aimed to investigate tendon cell responses to such cues in a cellular matrix enabling juxtacrine and paracrine signaling. Pro-inflammatory genes $I L-8, I L-1 \beta, T N F \alpha$, and IL- 6 showed increased expression in IL- $1 \beta$-magCSs, when com- pared with the other groups under study $(p<0.05)$ (Fig. 5Ai). Furthermore, the transcript levels of anti-inflammatory factors IL-10 and IL- 4 were decreased in IL- $1 \beta$-magCSs, in comparison to control (IL-10, $p<0.05 ;$ IL-4, $p<0.01$ ) and to PEMF stimulated (IL-10, $p<0.0001 ;$ IL-4, $p<0.0001$ ) conditions (Fig. 5Bi).

These results are supported by ELISA assays, which showed higher concentrations of TNF $\alpha$ and IL- 6 but lower amounts of IL10 released by IL- $1 \beta$-magCSs (TNF $\alpha, p<0.05$; IL-6, $p<0.0001$ in comparison to Control group). The effect of PEMF on the inflammatory profile of IL- $1 \beta$-magCSs was also investigated (Fig. 5B). PEMF stimulation caused a decrease in the genetic expression of pro-inflammatory cytokines and an increase in the expression of anti-inflammatory associated genes (IL-8, $p<0.001 ; I L-1 \beta, p<0.05$; TNF $\alpha, p<0.001 ;$ IL-6, $p<0.0001$ in comparison to IL- $\beta$-magCSs). 

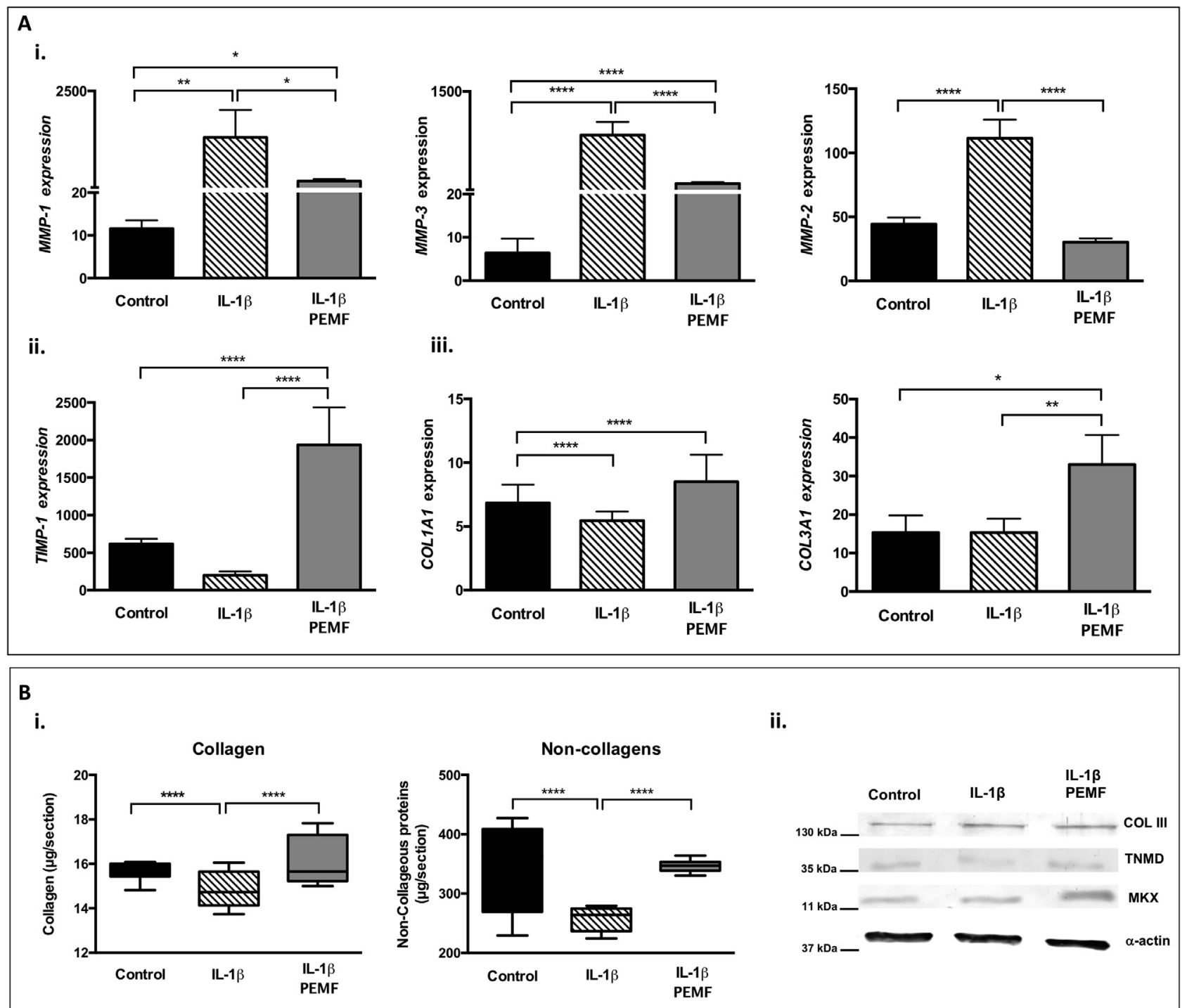

ii.

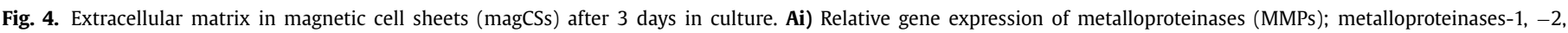

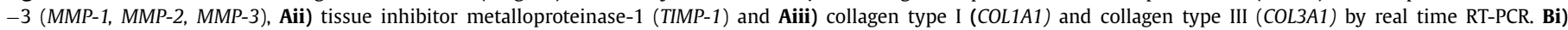

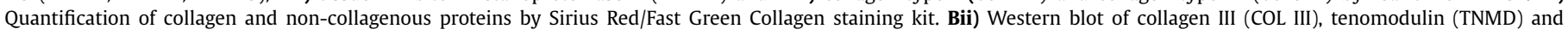

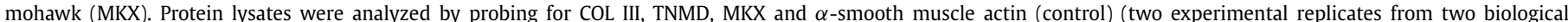

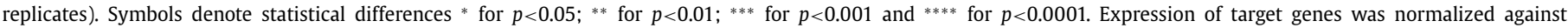

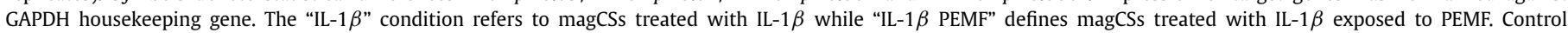
group refers to magCSs in the absence of IL- $1 \beta$ and PEMF.

Interestingly, the TNF $\alpha$ showed levels of expression even lower than the Control group $(p<0.05)$, while the expression of both IL-4 and $I L-10$ was approximately 3 -fold higher $(p<0.05)$ (Fig. $5 \mathrm{Bi})$. The dissimilar values between PEMF and Control groups may be associated to the anti-inflammatory effect of PEMF, even in the absence of IL- $1 \beta$ treatment.

Correlating with the results obtained for gene expression, the release into the culture medium of TNF $\alpha$ and IL- 6 were significantly reduced under PEMF in comparison to IL- $1 \beta$-magCSs $(p<0.0001)$ and to the Control group $(p<0.01)$ (Fig. 5Aii). The concentration of $\mathrm{PGE}_{2}$, a lipid mediator of pain and acute inflammation, also followed this trend (IL- $1 \beta$-magCSs, $p<0.001$; magCSs Control, $p<0.05)$ (Fig. 5Aii).

Additionally, the release of IL-10, was significantly increased with PEMF in comparison to the other conditions (Control group, $p<0.01$; IL- $1 \beta$-magCSs, $p<0.0001$ ) (Fig. 5Bii). As expected, tendon cells in magCSs constructions respond to IL- $1 \beta$ treatment with pronounced pro-inflammatory cues. Moreover, the pro- inflammatory signals can be modulated by exposure to PEMF, as observed in IL- $1 \beta$-magCSs, in which the expression levels of proinflammatory cues were lower than the ones shown for other conditions. According to these outcomes, PEMF seems to effectively reestablish the levels of anti-inflammatory factors in inflammatory conditions, necessary to the resolution of inflammation to continue healing.

\subsection{Involvement of MAPK signaling pathway in the regulation of inflammation cues}

In the complex inflammation process, different signaling pathways may be involved and may contribute to the activation and production of multiple pro-inflammatory molecules, namely IL$1 \beta$, TNF $\alpha$ or IL-6 [32-34]. The $\mathrm{p} 38$ mitogen associated protein kinase MAPK(ER1/2) pathway is involved in many tissues responses, including tendons tissues. [35-37]. In this sense, and considering the gene and protein response of magCSs to IL- $1 \beta$ treat- 

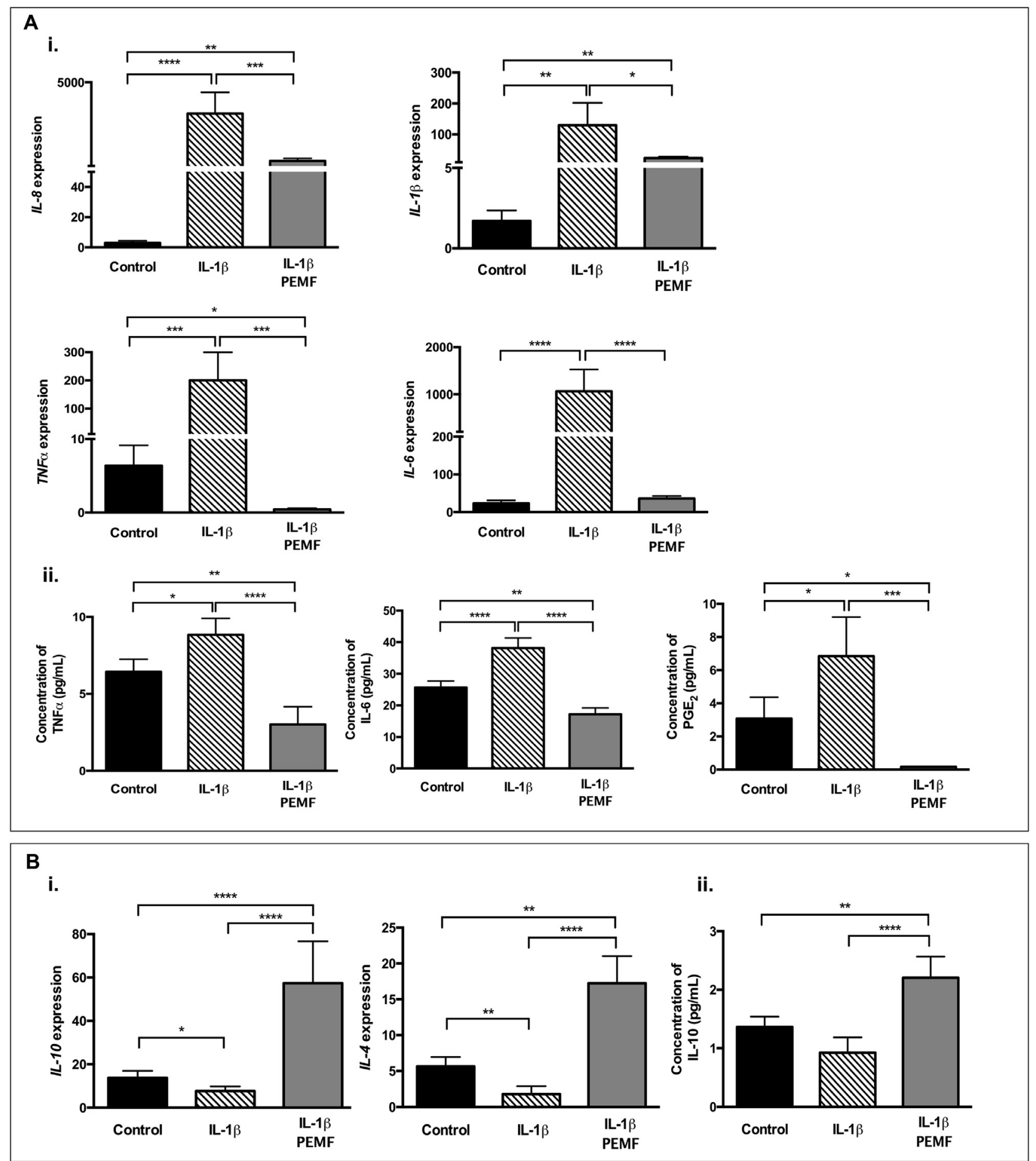

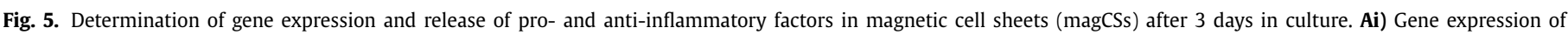

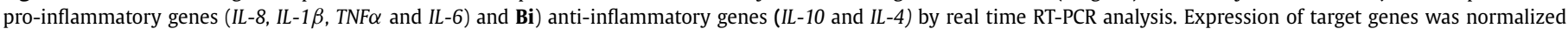

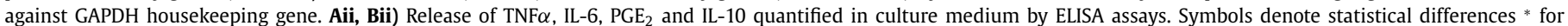

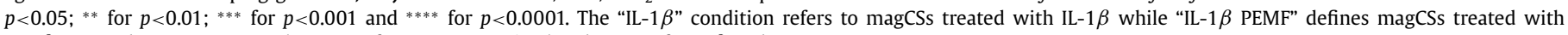
IL- $1 \beta$ exposed to PEMF. Control group refers to magCSs in the absence of IL- $1 \beta$ and PEMF.

ment concomitantly modulated by PEMF stimulation, we investigated the potential of MAPK/ERK1/2 on magCSs regulation (Fig. 6). The MAPK(ER1/2) activation is a consequence of different cytokine stimuli, including IL- $1 \beta$, triggering the expression of proinflammatory factors, such as COX-2 and $\mathrm{PGE}_{2}$ [38].
The results of phospho-MAPK(ERK1/2) were assessed by western blot (Fig. 6Ai). Although the bands do not show evident differences, the quantification of the bands intensity indicates that PEMF reduces phospho-MAPK(ERK1/2) in magCSs. Phosphorylated ERK1/2 was further assessed by ELISA assays and follow the trend 


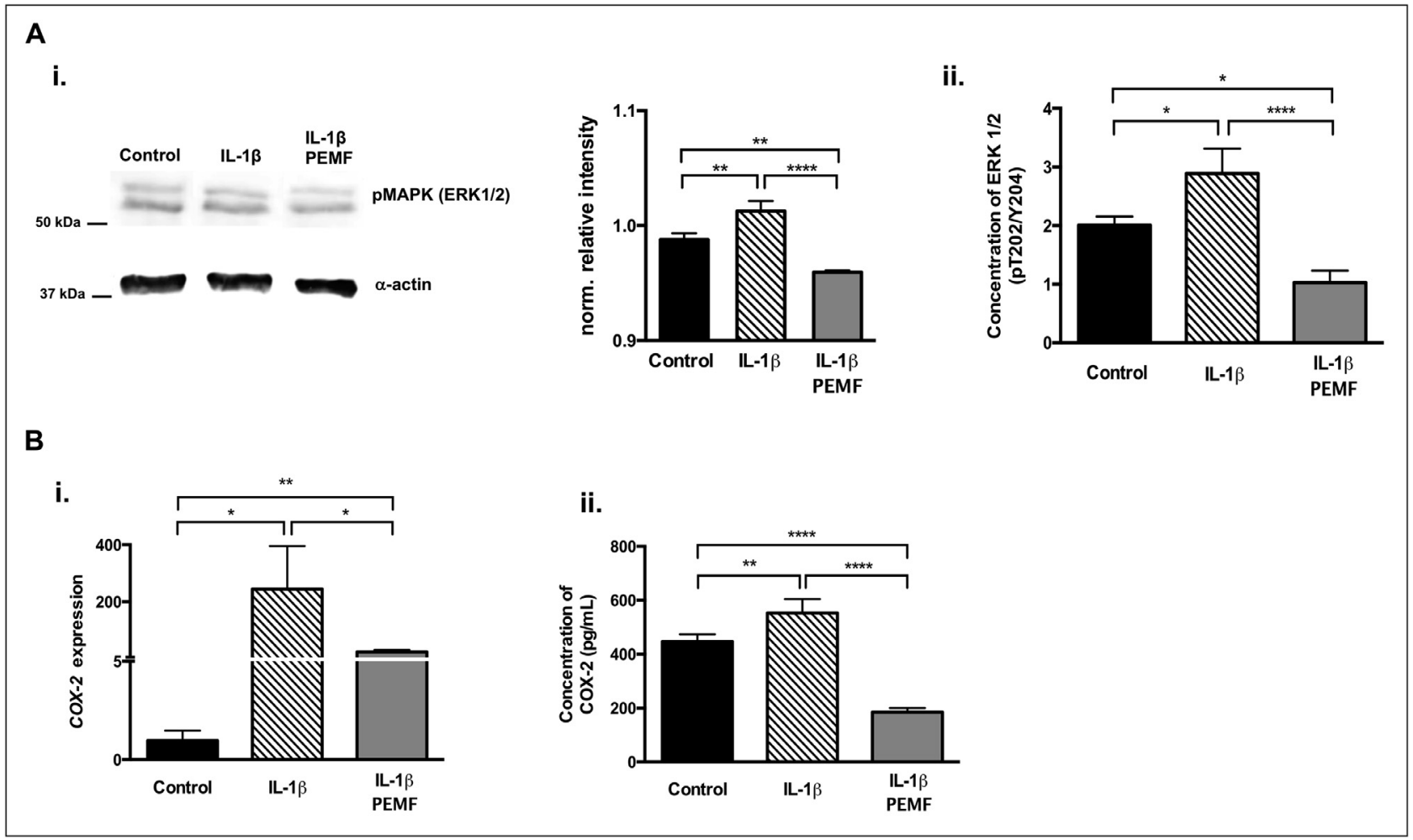

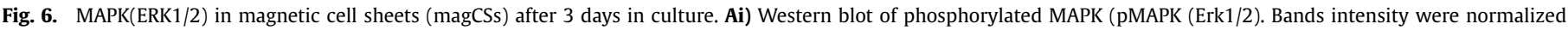

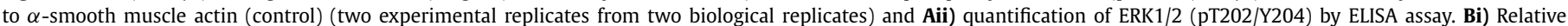

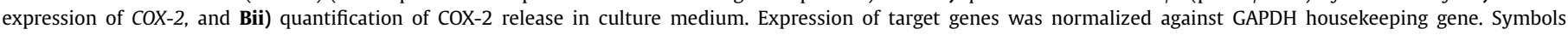

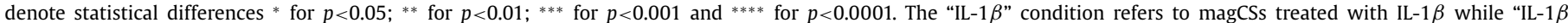
PEMF" defines magCSs treated with IL- $1 \beta$ exposed to PEMF. Control group refers to magCSs in the absence of IL- $1 \beta$ and PEMF.

of the cytokine profile, with an increased concentration in IL- $1 \beta$ magCSs (Control group, $p<0.05$ ) (Fig. 6Aii). Again, PEMF stimulated IL-1 $\beta$-magCSs show lower values in comparison to control group $(p<0.001)$ (Fig. 6Ai). These results suggest a possible role and targeted action of PEMF in MAPK(ERK1/2) activation in an IL- $\beta$ induced pro-inflammatory response.

The pro-inflammatory factors, COX-2 and $\mathrm{PGE}_{2}$, have been widely described as products resultant from MAPK pathway activation [37-39] (Fig. 6B). In this study, both COX-2 transcripts and protein expression were increased with IL- $1 \beta$ treatment $(p<0.05)$, and decreased with PEMF stimulation $(p<0.05)$ (Fig. 6Bi).

The release of $\mathrm{PGE}_{2}$ demonstrates a similar tendency (Fig. 6Bii). Interestingly, under PEMF the amounts of released $\mathrm{PGE}_{2}$ are lower than the ones of Control group $(p<0.05)$.

\section{Discussion}

\subsection{Establishment of an in vitro model with magCSs}

The prolonged exposure to pro-inflammatory mediators is known to lead to chronic inflammation [40], and ultimately to tendon disorders. Exposing magnetic tendon cell sheets to an inflammatory cytokine-rich milieu, we expected to more accurately predict tendon cell behavior in vivo and the potential immunomodulatory contributions of magCSs for tendon therapies.

To antagonize such influence and improve scaffold-free strategies, we also explored the immunomodulatory effect of PEMF on magCSs conditioned to IL- $1 \beta$.

The inflammatory stimulus provided by IL- $1 \beta$ influenced the tenogenic phenotype reducing the relative gene expression of $M K X$, $T N M D$, and $D C N$. Conversely, pro-inflammatory genes were increased, supported by the increment of pro-inflammatory medi- ators secreted by IL- $1 \beta$-magCSs. These outcomes, together with the decrease in anti-inflammatory genes and soluble factors, IL4 and IL-10, suggest that magCSs respond to IL- $1 \beta$ by increasing the expression and production of inflammatory factors into the environment $[41,42]$. This response also resulted in a decrease in COL1A1 expression and in the synthesis of ECM proteins (noncollagenous)suggesting that inflammatory stimuli may also interfere with the quality of the ECM. The matrix composition, organization and cell-matrix interactions are important for normal function of tendon. However, during tendinopathy conditions the normal composition of ECM suffers alterations and has been suggested to be the major factor for the development of this disorder [32]. These alterations interfere with the inflammatory cell signaling and with the cytokine release, thus affecting the important mechanisms of repair [43]. These mediators are responsible for ECM disorganization, which was recognized as a major cause for tendon injuries. In tendinopathy conditions, matrix degradation and the collagen content becomes more heterogeneous and disordered [31,44]. The degradation of collagen is normally regulated by MMPs and by the activity of their inhibitors (TIMPs). Miller et al. [45] reported that pro-inflammatory factors as TNF $\alpha$ and IL$1 \alpha$ stimulate catabolic degradation of collagen matrix via MMP-2 in intervertebral disk cells under normal and inflammatory environments, stimulated by IL- $1 \alpha$.

To elucidate on the mechanisms associated to the IL- $1 \beta$ treatment, we investigated a possible involvement of the intracellular signaling pathway, MAPK(ERK1/2), which is activated by stressful and pro-inflammatory stimuli.

Our results indicated that IL- $1 \beta$ treatment leads to the activation of MAPK(ERK1/2), likely due to the increment in IL- $1 \beta$ expression on magCSs. In addition, results demonstrated higher expres- 
sions of COX- 2 and $\mathrm{PGE}_{2}$ by the IL- $1 \beta$-magCSs. COX- 2 is responsible for the high levels of prostaglandins, such as $\mathrm{PGE}_{2}$ present in acute and chronic inflammation. MAPK (ERK1/2) signaling is involved in COX-2 expression and the inhibition of this pathway causes a reduced COX-2 expression [46]. Moreover, several studies focusing on inflammation reported that IL- $1 \beta$ influences the levels of COX-2 and $\mathrm{PGE}_{2}$ through the activation of the MAPK(ERK1/2) pathway [39,43-45,47].

\subsection{Contributions of PEMF stimulation to resolve inflammatory cues in IL-1 $\beta$-magCSs}

From our previous studies, PEMF has shown a great impact in the inflammatory profile of IL- $1 \beta$-hTDCs influencing the release and expression of cytokines $[17,18,39]$. Despite the fact that such works were performed in monolayer cultures and in the absence of MNPs, the combination of MNPs actuated by an external magnetic field could stimulate specific biochemical responses [48]. The response of MNPs to magnetic fields can provide additional signals that cells respond to, likely creating synergistic actions to guide the healing process [2]. For instance, the combination of MNPs and scaffolds with magnetic fields and stem cells has shown to increase the osteogenic differentiation, angiogenesis and bone regeneration [49]. Moreover, cell internalized MNPs can be visualized by conventional imaging techniques (e.g. MRI, CT), enabling visualization in preclinical and clinical approaches.

We investigated a possible influence of MNPs and PEMF on cell organization and distribution within magCSs. The results showed a tendency for parallel alignment of tendon cells, within magCSs constructions. The cellular organization is an important point to obtain proper healing and disorganized architectures may favor degenerative conditions mediated by pro-inflammatory niches [44].

In relation to gene expression level, magnetic stimulation in inflamed environment provoked a pronounced increase in $M K X$, $T N M D$ and $D C N$. Herein, the expression of genes encoding for matrix degrading enzymes were downregulated after the exposure to PEMF, and upregulated for the TIMP-1 in comparison to the Control group.

Moreover, the expression of collagens increased in IL- $1 \beta$ magCSs under PEMF stimulation and consequently the production of collagen proteins. During the healing process, high production of collagen fibers is essential, where collagen type III is required for the assemble of collagen type I fibers. Thus, an increase in collagen type III may suggest cellular guidance towards healing [31].

Our results demonstrate that PEMF supports the production of a tendon-like matrix, promotes collagen synthesis, and a decrease in MMPs gene expression, evidencing a regulatory influence of PEMF in tendon healing process. Understanding the mechanisms associated to the expression of inflammatory mediators may provide molecular tools for improving current therapeutics [50]. The effect of PEMF in MAPK(ERK1/2) activation of IL- $1 \beta$-magCSs and the consequent synthesis of COX-2 and $\mathrm{PGE}_{2}$, was investigated in this work. Several studies have reported that PEMF can modulate the activation of intracellular signaling associated to inflammatory profiles mediated by NF-Kb, mTOR or MAPK pathways [17,51]. Additionally, the phosphorylation of p38 MAPK(ERK1/2) was shown to be inhibited by the exposure to PEMF $(1.5 \mathrm{mT}, 75 \mathrm{~Hz}, 10 \%$ duty cycle) in an inflammatory model of synovitis, leading to the decrease of TNF $\alpha$ [52].

In our study, we demonstrate not only a reduction on the phosphorylation of MAPK(ERK1/2) in IL- $1 \beta$-magCSs but also reduced levels of COX-2 and $\mathrm{PGE}_{2}$. An exposure to a PEMF $(1.5 \mathrm{mT}, 75 \mathrm{~Hz}$, $1 / 10$ duty cycle) was also shown to reduce the release of $\mathrm{PGE}_{2}$ as well as of IL- 6 and IL- 8 from bovine synovial fibroblasts from osteoarthritis patients treated with IL- $\beta$ [53]. Thus, the reduction on the phosphorylation of MAPK(ERK1/2) could also indicate that PEMF may influence the expression and synthesis of proinflammatory cytokines and lipid mediators COX-2 and $\mathrm{PGE}_{2}$ in magCSs through MAPK(ERK1/2). These outcomes are in accordance with published studies [51] supporting the application of magnetic fields in pathologies with an inflammatory context.

Overall, these results show the applicability of magCSs as a cellular model for tendon studies. Moreover, PEMF shows a relevant influence over inflammatory mediators and in the quality and quantity of ECM in magCSs that goes beyond the expression of cytokines, and may depend on MAPK(ERK1/2) pathway.

\section{Conclusions}

This work shows that magCSs technologies offer new possibilities as advanced cell culture models for tendon tissue engineering, including pathophysiological models of inflammation to assist the understanding of the onset and progression of tendon disorders. In accordance to previous studies, PEMF evidences a modulatory action in the inflammatory profile of IL- $\beta$ primed tendon cells in magCSs constructions, via MAPK(ERK1/2) pathway. The remote control over magCSs strengthens the role of PEMF in tendon therapies, and highlights the promise of magCSs as a living patch to overcome sustained inflammatory events and to drive tendon repair and regeneration.

\section{Data availability statement}

The data that support the findings of this study are available from the corresponding authors upon reasonable request.

\section{Declaration of Competing Interest}

The authors declare that they have no known competing financial interests or personal relationships that could have appeared to influence the work reported in this paper.

\section{Acknowledgements}

This research was funded by the ERC CoG MagTendon (No. 772817), Fundação para a Ciência e Tecnologia (FCT) for the doctoral grant PD/BD/128089/2016 of A. Vinhas and the project MagTT PTDC/CTM-CTM/29930/2017 (POCI-01-0145-FEDER-29930), project Norte-01-0145-FEDER-02219015 supported by Norte Portugal Regional Operational Programme (NORTE 2020) and EC Twinning project Achilles (No. 810850). The authors thank Hospital da Prelada (Porto, Portugal) for providing tendon samples.

\section{Supplementary materials}

Supplementary material associated with this article can be found, in the online version, at doi:10.1016/j.actbio.2021.06.036.

\section{References}

[1] D. Cigognini, A. Lomas, P. Kumar, A. Satyam, A. English, A. Azeem, A. Pandit, D. Zeugolis, Engineering in vitro microenvironments for cell based therapies and drug discovery, Drug Discov. Today 18 (21-22) (2013) 1099-1108.

[2] A.I. Gonçalves, M.T. Rodrigues, M.E. Gomes, Tissue-engineered magnetic cell sheet patches for advanced strategies in tendon regeneration, Acta Biomater. 63 (2017) 110-122.

[3] Y. Lu, W. Zhang, J. Wang, G. Yang, S. Yin, T. Tang, C. Yu, X. Jiang, Recent advances in cell sheet technology for bone and cartilage regeneration: from preparation to application, Int. J. Oral Sci. 11 (2) (2019) 17.

[4] M. Li, J. Ma, Y. Gao, L. Yang, Cell sheet technology: a promising strategy in regenerative medicine, Cytotherapy 21 (1) (2019) 3-16.

[5] Y. Qi, W. Yan, Mesenchymal stem cell sheet encapsulated cartilage debris provides great potential for cartilage defects repair in osteoarthritis, Med. Hypotheses 79 (3) (2012) 420-421. 
[6] H. Shen, R. Jayaram, S. Yoneda, S.W. Linderman, S.E. Sakiyama-Elbert, Y. Xia, R.H. Gelberman, S. Thomopoulos, The effect of adipose-derived stem cell sheets and CTGF on early flexor tendon healing in a canine model, Sci. Rep. 8 (1) (2018) 11078

[7] A. Ito, M. Hayashida, H. Honda, K. Hata, H. Kagami, M. Ueda, T. Kobayashi, Construction and harvest of multilayered keratinocyte sheets using magnetite nanoparticles and magnetic force, Tissue Eng. 10 (5(6)) (2004) 873-880.

[8] A.I. Goncalves, M.S. Miranda, M.T. Rodrigues, R.L. Reis, M.E. Gomes, Magnetic responsive cell-based strategies for diagnostics and therapeutics, Biomed. Mater. 13 (5) (2018) 054001.

[9] H. Akiyama, A. Ito, Y. Kawabe, M. Kamihira, Genetically engineered angiogenic cell sheets using magnetic force-based gene delivery and tissue fabrication techniques, Biomaterials 31 (6) (2010) 1251-1259.

[10] M. Ishii, R. Shibata, Y. Numaguchi, T. Kito, H. Suzuki, K. Shimizu, A. Ito, H. Honda, T. Murohara, Enhanced angiogenesis by transplantation of mesenchymal stem cell sheet created by a novel magnetic tissue engineering method, Arterioscler. Thromb. Vasc. Biol. 31 (10) (2011) 2210-2215.

[11] H. Akiyama, A. Ito, M. Sato, Y. Kawabe, M. Kamihira, Construction of cardiac tissue rings using a magnetic tissue fabrication technique, Int. J. Mol. Sci. 11 (8) (2010) 2910-2920

[12] K. Shimizu, A. Ito, T. Yoshida, Y. Yamada, M. Ueda, H. Honda, Bone tissue engineering with human mesenchymal stem cell sheets constructed using magnetite nanoparticles and magnetic force, J. Biomed. Mater. Res. B Appl. Biomater. 82 (2) (2007) 471-480.

[13] Y. Yamamoto, A. Ito, M. Kato, Y. Kawabe, K. Shimizu, H. Fujita, E. Nagamori, M. Kamihira, Preparation of artificial skeletal muscle tissues by a magnetic force-based tissue engineering technique, J. Biosci. Bioeng. 108 (6) (2009) $538-543$.

[14] C. Tang, Y. Chen, J. Huang, K. Zhao, X. Chen, Z. Yin, B.C. Heng, W. Chen, W. Shen, The roles of inflammatory mediators and immunocytes in tendinopathy, J Orthop Translat 14 (2018) 23-33.

[15] K. Ren, R. Torres, Role of interleukin-1beta during pain and inflammation, Brain Res. Rev. 60 (1) (2009) 57-64.

[16] C.A. Dinarello, Interleukin-1 in the pathogenesis and treatment of inflammatory diseases, Blood 117 (14) (2011) 3720-3732.

[17] A. Vinhas, M.T. Rodrigues, A.I. Goncalves, R.L. Reis, M.E. Gomes, Pulsed Electromagnetic Field Modulates Tendon Cells Response in IL-1beta-Conditioned Environment, J. Orthop. Res. 38 (1) (2019) 160-172.

[18] A. Vinhas, A.F. Almeida, A.I. Goncalves, M.T. Rodrigues, M.E. Gomes, Magnetic stimulation drives macrophage polarization in cell to-cell communication with IL-1beta primed tendon cells, Int. J. Mol. Sci. 21 (15) (2020).

[19] R. Gehwolf, B. Schwemberger, M. Jessen, S. Korntner, A. Wagner, C. Lehner, N. Weissenbacher, H. Tempfer, A. Traweger, Global responses of il-1beta-primed 3d tendon constructs to treatment with pulsed electromagnetic fields, Cells 8 (5) (2019).

[20] L. de Girolamo, D. Stanco, E. Galliera, M. Vigano, A. Colombini, S. Setti, E. Vianello, M.M. Corsi Romanelli, V. Sansone, Low frequency pulsed electromagnetic field affects proliferation, tissue-specific gene expression, and cytokines release of human tendon cells, Cell Biochem. Biophys. 66 (3) (2013) 697-708.

[21] C.L. Ross, B.S. Harrison, Effect of pulsed electromagnetic field on inflammatory pathway markers in RAW 264.7 murine macrophages, J. Inflamm. Res. 6 (2013) 45-51.

[22] M. Nardoni, E.Della Valle, M. Liberti, M. Relucenti, M.A. Casadei, P. Paolicelli, F. Apollonio, S. Petralito, Can pulsed electromagnetic fields trigger on-demand drug release from high-Tm Magnetoliposomes? Nanomaterials (Basel) 8 (4) (2018).

[23] G. Rahmi, L. Pidial, A.K. Silva, E. Blondiaux, B. Meresse, F. Gazeau, G. Autret, D. Balvay, C.A. Cuenod, S. Perretta, B. Tavitian, C. Wilhelm, C. Cellier, O. Clement, Designing 3D mesenchymal stem cell sheets merging magnetic and fluorescent features: when cell sheet technology meets image-guided cell therapy, Theranostics 6 (5) (2016) 739-751.

[24] A.I. Gonçalves, R. Costa-Almeida, P. Gershovich, M.T. Rodrigues, R.L. Reis, M.E. Gomes, Cell-Based Approaches for Tendon Regeneration, in: M.E. Gomes, R.L. Reis, M.T. Rodrigues (Eds.), Tendon Regeneration: Understanding tissue Physiology and Development to Engineer Functional Substitutes, Elsevier, 2015, pp. 187-203.

[25] R. Costa-Almeida, L. Gasperini, J. Borges, P.S. Babo, M.T. Rodrigues, J.F. Mano, R.L. Reis, M.E. Gomes, Microengineered Multicomponent Hydrogel Fibers: combining Polyelectrolyte Complexation and Microfluidics, Acs Biomater. Sci. Eng. 3 (7) (2017) 1322-1331.

[26] Y. Bi, D. Ehirchiou, T.M. Kilts, C.A. Inkson, M.C. Embree, W. Sonoyama, L. Li, A.I. Leet, B.M. Seo, L. Zhang, S. Shi, M.F. Young, Identification of tendon stem/progenitor cells and the role of the extracellular matrix in their niche, Nat. Med. 13 (10) (2007) 1219-1227.

[27] A. Vinhas, M.T. Rodrigues, A.I. Gonçalves, R.L. Reis, M.E. Gomes, Magnetic responsive materials modulate the inflammatory profile of IL- $1 \beta$ conditioned tendon cells, Acta Biomater. (2020).

[28] K. Zhang, S. Asai, B. Yu, M. Enomoto-Iwamoto, IL-1beta irreversibly inhibits tenogenic differentiation and alters metabolism in injured tendon-derived progenitor cells in vitro, Biochem. Biophys. Res. Commun. 463 (4) (2015) 667-672.
[29] J.D. Rees, M. Stride, A. Scott, Tendons-time to revisit inflammation, Br. J. Sports Med. 48 (21) (2014) 1553-1557.

[30] M. Raeeszadeh-Sarmazdeh, L.D. Do, B.G. Hritz, Metalloproteinases and Their Inhibitors: potential for the Development of New Therapeutics, Cells 9 (5) (2020).

[31] H.R. Screen, D.E. Berk, K.E. Kadler, F. Ramirez, M.F. Young, Tendon functional extracellular matrix, J. Orthop. Res. 33 (6) (2015) 793-799.

[32] K. Lipman, C. Wang, K. Ting, C. Soo, Z. Zheng, Tendinopathy: injury, repair, and current exploration, Drug Des. Devel. Ther. 12 (2018) 591-603.

[33] L. Chen, H. Deng, H. Cui, J. Fang, Z. Zuo, J. Deng, Y. Li, X. Wang, L. Zhao, Inflammatory responses and inflammation-associated diseases in organs, Oncotraget 9 (2018) 7204-7218.

[34] A. Kulawik, R. Engesser, C. Ehlting, A. Raue, U. Albrecht, B. Hahn, W.D. Lehmann, M. Gaestel, U. Klingmuller, D. Haussinger, J. Timmer, J.G. Bode, IL-1beta-induced and p38(MAPK)-dependent activation of the mitogen-activated protein kinase-activated protein kinase 2 (MK2) in hepatocytes: signal transduction with robust and concentration-independent signal amplification, J. Biol. Chem. 292 (15) (2017) 6291-6302.

[35] J.M. Wilde, J.P. Gumucio, J.A. Grekin, D.C. Sarver, A.C. Noah, D.G. Ruehlmann, M.E. Davis, A. Bedi, C.L. Mendias, Inhibition of p38 mitogen-activated protein kinase signaling reduces fibrosis and lipid accumulation after rotator cuff repair, J. Shoulder Elbow Surg. 25 (9) (2016) 1501-1508.

[36] T. Nishikai-Yan Shen, S. Kanazawa, M. Kado, K. Okada, L. Luo, A. Hayashi, H. Mizuno, R. Tanaka, Interleukin-6 stimulates Akt and p38 MAPK phosphorylation and fibroblast migration in non-diabetic but not diabetic mice, PLoS One 12 (5) (2017) e0178232.

[37] W. Liu, S. Huang, Y. Li, Y. Li, D. Li, P. Wu, Q. Wang, X. Zheng, K. Zhang, Glycyrrhizic acid from licorice down-regulates inflammatory responses via blocking MAPK and PI3K/Akt-dependent NF-kappaB signalling pathways in TPA-induced skin inflammation, Medchemcomm 9 (9) (2018) 1502-1510.

[38] E. Molina-Holgado, S. Ortiz, F. Molina-Holgado, C. Guaza, Induction of COX-2 and PGE2 biosynthesis by IL-1b is mediated by PKC and mitogen-activated protein kinases in murine astrocytes, Br. J. Pharmacol. 131 (2000) 152-159.

[39] Q. Xia, Q. Hu, H. Wang, H. Yang, F. Gao, H. Ren, D. Chen, C. Fu, L. Zheng, X. Zhen, Z. Ying, G. Wang, Induction of COX-2-PGE2 synthesis by activation of the MAPK/ERK pathway contributes to neuronal death triggered by TDP-43-depleted microglia, Cell Death. Dis. 6 (2015) e1702.

[40] M.A. Sugimoto, L.P. Sousa, V. Pinho, M. Perretti, M.M. Teixeira, Resolution of Inflammation: what Controls Its Onset? Front. Immunol. 7 (2016) 160.

[41] N.L. Millar, G.A. Murrell, I.B. McInnes, Inflammatory mechanisms in tendinopathy - towards translation, Nat. Rev. Rheumatol. 13 (2) (2017) 110-122.

[42] W. Morita, S.G. Dakin, S.J.B. Snelling, A.J. Carr, Cytokines in tendon disease, Bone Joint Res. 6 (12) (2017) 656-664.

[43] F.G. Thankam, Z.K. Roesch, M.F. Dilisio, M.M. Radwan, A. Kovilam, R.M. Gross, D.K. Agrawal, Association of Inflammatory Responses and ECM Disorganization with HMGB1 Upregulation and NLRP3 Inflammasome Activation in the Injured Rotator Cuff Tendon, Sci. Rep. 8 (1) (2018) 8918.

[44] A.D. Schoenenberger, J. Foolen, P. Moor, U. Silvan, J.G. Snedeker, Substrate fiber alignment mediates tendon cell response to inflammatory signaling, Acta Biomater. 71 (2018) 306-317.

[45] S.L. Miller, D.G. Coughlin, E.I. Waldorff, J.T. Ryaby, J.C. Lotz, Pulsed electromagnetic field (PEMF) treatment reduces expression of genes associated with disc degeneration in human intervertebral disc cells, Spine J. 16 (6) (2016) 770-776.

[46] W. Cho, J. Choe, Prostaglandin E2 stimulates COX-2 expression via mitogen-activated protein kinase p38 but not ERK in human follicular dendritic cell-like cells, BMC Immunol. 21 (1) (2020) 20.

[47] S.J. Desai, B. Prickril, A. Rasooly, Mechanisms of Phytonutrient Modulation of Cyclooxygenase-2 (COX-2) and Inflammation Related to Cancer, Nutr. Cancer 70 (3) (2018) 350-375.

[48] J. Henstock, A. El Haj, Controlled mechanotransduction in therapeutic MSCscan remotely controlled magnetic nanoparticles regenerate bones? Future Med. (2015).

[49] Y. Xia, J. Sun, L. Zhao, F. Zhang, X.J. Liang, Y. Guo, M.D. Weir, M.A. Reynolds, N. Gu, H.H.K. Xu, Magnetic field and nano-scaffolds with stem cells to enhance bone regeneration, Biomaterials 183 (2018) 151-170.

[50] J.D. O'Neil, A.J. Ammit, A.R. Clark, MAPK p38 regulates inflammatory gene expression via tristetraprolin: doing good by stealth, Int. J. Biochem. Cell Biol. 94 (2018) 6-9

[51] C.L. Ross, Y. Zhou, C.E. McCall, S. Soker, T.L. Criswell, The Use of Pulsed Electromagnetic Field to Modulate Inflammation and Improve Tissue Regeneration: a Review, Bioelectricity 1 (4) (2019) 247-259.

[52] J. Ouyang, B. Zhang, L. Kuang, P. Yang, X. Du, H. Qi, N. Su, M. Jin, J. Yang, Y. Xie, Q. Tan, H. Chen, S. Chen, W. Jiang, M. Liu, X. Luo, M. He, Z. Ni, L. Chen, Pulsed Electromagnetic Field Inhibits Synovitis via Enhancing the Efferocytosis of Macrophages, Biomed. Res. Int. 2020 (2020) 4307385.

[53] A. Ongaro, K. Varani, F.F. Masieri, A. Pellati, L. Massari, R. Cadossi, F. Vincenzi, P.A. Borea, M. Fini, A. Caruso, M. De Mattei, Electromagnetic fields (EMFs) and adenosine receptors modulate prostaglandin $\mathrm{E}(2)$ and cytokine release in human osteoarthritic synovial fibroblasts, J. Cell. Physiol. 227 (6) (2012) 2461-2469. 